\title{
Multiple sclerosis: Pathology, diagnosis and treatments (Review)
}

\author{
WEN-JUAN HUANG, WEI-WEI CHEN and XIA ZHANG \\ Department of Neurology, Xuzhou Central Hospital, Xuzhou, Jiangsu 221009, P.R. China
}

Received July 22, 2016; Accepted March 27, 2017

DOI: $10.3892 /$ etm.2017.4410

\begin{abstract}
Multiple sclerosis (MS) is a complex neurodegenerative disease affecting the central nervous system (CNS). The onset of MS has been typically observed in individuals aged from 20 to 40 -years, with the female to male ratio of 1:2. MS appears as abrupt onset of focal sensory disturbances that is accompanied by unilateral painless damage of vision, double vision, limb weakness, unsteadiness of gait, and bowel or bladder symptoms. Whereas the exact etiology of the disease is unknown, observational research has suggested genetic and environment influences through an underlined pathophysiology widely believed to be autoimmune in nature. Indeed, plaque of demyelination inside of the CNS with relative conservation of axons remains the clinical symptoms of MS. However, considerable advances in understanding the pathology have contributed to an early diagnosis, particularly the exact neuroanatomical setting of plaques. Accordingly, magnetic resonance imaging has been considered as the primarily adjunctive modality for the constant detection of abnormal white matter. In addition, the analysis of cerebrospinal fluid contents has also been of interest for the diagnosis to discriminate other affections such infection or vasculitis. These resulted in a broad variety of therapies that considerably control the activity and change the course and prognosis of the disease. In the present review, we evaluate the current state of knowledge on MS with emphasis on the pathology itself, the diagnosis and common therapeutical approaches accurately used.
\end{abstract}

\section{Contents}

1. Introduction

2. Pathology and diagnosis

3. Treatments

4. Conclusion

Correspondence to: Dr Wen-Juan Huang, Department of Neurology, Xuzhou Central Hospital, 199 Jiefang South Road, Xuzhou, Jiangsu 221009, P.R. China

E-mail: ftmlb8556215@163.com

Key words: multiple sclerosis, pathophysiology, treatment options, diagnostic criteria

\section{Introduction}

Multiple sclerosis (MS) is a chronic complex neurodegenerative disease, targeting the central nervous system (CNS) and widely believed to be autoimmune in nature. it is mediated by autoreactive lymphocytes that cross the blood-brain barrier (BBB) and enter the CNS where they cause local inflammation that results in demyelination, gliotic scarring, and axonal loss (1).

Approximately, 2.5 million individuals are affected worldwide, and young individuals aged between 20- and 40-years are mainly affected (2). The higher frequency of MS is seen in women who are affected twice as often as men (3).

However, advances in the understanding of genetics of MS have been relatively slow up until the last decade, since the discovery of the importance of variants in HLA genes of the major histocompatibility complex (MHC) in the 1970s (4). Accordingly, genome-wide association studies (5) and the International MS Genetics Consortium (IMSGC) (6) have opened up an entire new field of research into genetic and subtle epigenetic influences.

However, MS is likely influenced by B cells through a variety of mechanisms, including the establishment of ectopic lymphoid follicles within the CNS, antigen presentation, cytokine production and antibody production (7). In general, the affected individuals initially present a relapsing-remitting disease course that is followed by a progressive phase several years later (8). The underlying pathophysiology hallmarks are inflammatory lesions that result in neuronal demyelination, axonal damage and subsequent neurological dysfunctions following the formation of multiple plaques in the grey and white matter of the brain and spinal cord (9). Accordingly, MS is considered the most common cause of neurological disability since inflammatory lesions associated with MS can affect a large range of systems to a variable degree and cause a myriad of neurological symptoms and comorbidities. These include sensory loss, visual disturbance, double vision, muscle weakness, ataxia and impaired balance, which may considerably reduce the quality of life in affected individuals $(10,11)$. The exact clinical symptoms are the result of plaques of demyelination within the CNS with relative preservation of the axons. Indeed, the myelin sheath around axons is crucial for the transmission of information between regions within the CNS.

Thus, the clinical symptoms of MS are determined by the exact neuroanatomical location of the plaque; the disease being essentially diagnosed by the manifestation of symptoms and 
signs attributable to lesions of white matter, and the diagnosis is supported by laboratory tests combined with the exclusion of conditions that mimic MS. A poor prognosis of diagnosed patients is generally associated with factors such as older age at onset and a greater number of relapses during the first few years. Although the disease remains incurable, several therapies currently approved are able of modify disease course and improving the quality of life for patients. These therapies are discussed in the current review. We first briefly present the clinical course of MS, giving an overview of the status of knowledge regarding the pathology and diagnosis of this disabling disease to better understand the rational design of therapeutic strategies.

\section{Pathology and diagnosis}

Clinical course of MS. Patients suffering from MS show a wide variety of neurological symptoms that originate from different parts of the CNS. They can occur alone or combined. According to the spatial distribution of the lesions in the CNS, the symptoms are quite variable from patient to patient, but commonly involve sensory disturbance, bladder dysfunction, cognitive deficits, unilateral painless loss of vision, double vision, limb weakness, ataxia, fatigue, and bowel troubles (12). Although MS is associated with change in average life expectancy, it is not considered a fatal condition and clinical course, which appears highly variable, is unpredictable for the individual patient. Typically, the disease starts with the sudden onset of neurological deficits. In the majority of cases, the disease shows a purely relapsing remitting course with the absence of symptoms except for the relapses. Specifically, the recurrent periods are followed by remission phases after complete or partial recovery (13). Over time, most cases evolve into a secondary progressive form of disease, which is characterized by continuous irreversible neurological impairment between relapses. Only a small percentage of patients experience a gradually progressive clinical course from the onset of the disease (14). Such a clinical course refers to the principal progressive form of MS, in which symptoms start and worsen insidiously with no periods of remission. Therefore, the steady progression of symptoms associated with primary progressive MS leads to disability from the beginning. Patients suffering from this form of MS do not show a female prevalence, and begin the disease later than those with the relapsing remitting form (15).

Pathology. MS refers to the plaques that form in the CNS combined with inflammation, demyelination, axonal injury and axonal loss. These plaques are found in the brain and spinal cord, essentially in the white matter around the ventricles, optic nerves and tracts, corpus callosum, cerebellar peduncles, long tracts and subpial region of the spinal cord and brainstem, but also in the gray matter (16). They are expressed in all forms of MS, but vary over time quantitatively and qualitatively showing a profound heterogeneity in the structure and immunopathological patterns of demyelination and oligodendrocyte pathology between relapsing remitting course and progressive forms of disease (17).

During the early stages of the relapsing remitting course, the pathology is marked by important demyelination and a variable degree of axonal loss and reactive gliosis (18). Patients in general, present with focal inflammatory plaques that contain demyelinated axons, reduced number of oligodendrocytes, astrocyte proliferation with subsequent gliosis, transected axons, and perivenular as well as parenchymal infiltrates of lymphocytes and macrophages. In the progressive course, MS is dominated by diffuse gray and white matter atrophy and characterized by low-grade inflammation and microglial activation at the plaque borders combined with diffuse injury of the normal-appearing white matter outside the plaque (19). Inflammation, microglial activation, axonal and myelin injury occurring during this course are followed by secondary demyelination (20). In general, the patterns of tissue injured in patients presented with primary or secondary progressive course of MS are homogeneous. They showed oligodendrocyte loss, preferential destruction of small-caliber axons, astrocytic gliosis, and demyelination that consists of the essential criteria (21). Demyelination and subsequent neurodegeneration associated with different forms of MS involved various components of adaptive and innate immunity (22). Myelin sheaths are particularly vulnerable to non-specific products, such as cytotoxic cytokines, excitotoxins, reactive oxygen or nitric oxide species, which are released by activated macrophages and microglia (23). However, the most commonly observed patterns of demyelination are antibody and complement-associated changes, as well as hypoxia-like tissue injury, in which the initiation of demyelination is attributed to the degeneration of distal oligodendrocyte processes and apoptosis of oligocytes, while the loss of polarity by astrocytes leads to the disturbance of the structural organizational of the perivascular glia limitans (24).

Classically, MS is regarded as a T cell-mediated autoimmune disorder with a predominance of $\mathrm{CD}^{+}$cells compared with other T-cell subsets, B cells or plasma cells. It is believed that this disease begins in inflammatory-induced lesions consisting mainly of $\mathrm{CD} 8^{+} \mathrm{T}$ cells, and $\mathrm{CD} 4^{+} \mathrm{T}$ cells, and activate microglia/macrophages $(25,26)$.

Evidence of the suppression of function that restricts $\mathrm{CD} 4{ }^{+} \mathrm{T}$-cell responses and the tissue-damaging role of $\mathrm{CD}^{+} \mathrm{T}$ cells reported to co-localize with axonal pathology have been observed $(27,28)$. Indeed, the specific interaction of $\mathrm{CD}^{+} \mathrm{T}$ cells with target cells requires $\mathrm{MHC}-\mathrm{I}$ expression which is tightly regulated in neurons and MHC-I molecules only in response to strong danger signals such as proinflammatory cytokines IFN- $\gamma$ or TNF- $\alpha$ (29).

Diagnosis. The diagnosis of MS is primary clinical and is dependent on the demonstration of neurologic signs and symptoms subsequent to white matter lesions. To distinguish MS from other conditions with similar neurologic manifestations, several criteria $(30,31)$ including McDonald criteria (21) have been proposed. These criteria depend on the demonstration of lesions disseminated in time and space to exclude alternative diagnoses. The requirement for such dissemination of lesions is achieved with adjuvant laboratory tests and imaging, including magnetic resonance imaging (MRI) of brain and spinal cord, cerebrospinal fluid analysis, and functional assays of the nervous system. The McDonald criteria, which combine these paraclinical assessments with 
clinical examination, are the most commonly used diagnostic approach. Currently, the diagnosis of MS depends largely on the results of MRI examination. Using gadolinium as a contrast agent to highlight active plaques, MRI allows detecting plaques that are ongoing to destruction of the BBB, and also those not associated with neurological symptoms at the time of the assessment. Therefore, relapsing remitting MS can be diagnosed earliest after a single relapse with an MRI scan showing gadolinium-enhancing and non-enhancing lesions disseminated in space. Indeed, since 2014 a published classification of the clinically subtypes of MS (32) has been established by the International Advisory Committee on Clinical Trials. In this new classification, MRI activity (gadolinium-enhancing lesions and new or unequivocally enlarging T2 lesions) and clinical relapses have been taken into account. Nowadays, MS patients are classified as either progressive MS that is divided into either primary progressive or secondary progressive with phenotype defined as either active (clinically or radiologically) or inactive. According to committee recommendations, patients with relapsing MS must share a clinical assessment and MRI brain at least annually, although this may be a significant burden for some radiology departments.

\section{Treatments}

Treatment of MS is challenging and involves several drugs acting via different mechanisms. The indication essentially depends on the clinical course and form of the disease. Although there is no proven therapy for the primary progressive form, several drugs are available to occasionally ameliorate the secondary progressive form and beneficially modify the activity of disease when dominated by the relapsing-remitting course. Indeed, since the introduction of disease-modifying therapies in the 1990 s, the number of agents used in the relapsing form of MS has considerably grown. Over 10 products, varying in their efficiency, side-effect profile and safety security requirements, have been approved, and several more are anticipated.

There are actually 12 products licenced by The European Medicines Agency (EMA) and U.S. Food and Drug Administration (FDA). Among them dimethyl fumarate (Tecfidera), alemtuzumab (Lemtrada), pegylated interferon- $\beta$ (Plegridy) and glatiramer acetate (Copaxone) $40 \mathrm{mg}$ have been produced and licenced since 2013.

Among drugs currently available, Interferon- $\beta$-1a (Avonex) variants and Glatiramer acetate have been utilized for more than two decades, and are used as first-line therapies for the relapsing-remitting form of MS (33). They efficiently reduce the relapse rate, ameliorate relapse severity and delay the progression of neurological disability.

Dimethyl fumarate (Tecfidera), originally used in the treatment of psoriasis, has shown proven effects in clinical trials. It reduces the MS annualised relapse rate by approximately $50 \%(34,35)$.

However, vitamin D has provided beneficial therapeutic effects in small studies (36-41).

Another compound that may be comsidered beneficial for MS is Simvastatin.

Indeed, in a primary outcome measure of the rate of whole brain atrophy, with additional secondary outcomes both MRI (new or enlarging T2 lesions) and clinical, expanded disability status scale (EDSS), MS functional composite scale (MSFC), MS impact scale-29 (MSIS-29) and relapse frequency were measured in a phase 2-clinical trial (42). The study emphasized beneficial effects of EDSS and MSIS-29, but not MSFC. In the MS-SMART study, the Simvastatin trial methodology currently being further tested in exploratory studies repurposing other agents (fluoxetine, amiloride and riluzole) that may have neuroprotective properties (43).

Of note, Daclizumab, a humanized antibody against IL2R $\alpha$ chain has been shown to strongly reduce the brain inflammation in MS patients thereby leading to only a mild functional blockade of CD4 T cells, which is known as the major candidate in MS pathogenesis (44). Another compound that may have a promising future is Ocrelizumab, and anti-CD20 monoclonal antibody targeting B cells that appears to be efficacious and well tolerated. Ocrelizumab reduces relapses by nearly $50 \%$ compared to interferon- $\beta$ in two phase 3 trials (OPERA I and II) (45). The development of famciclovir, a vaccine to prevent Epstein-Barr virus infection is a key research interest in MS patients (46).

\section{Conclusion}

Although MS remains an untreatable disease, the currently used active treatments of the relapsing-remitting phase, not only provide higher choice to physicians and patients, but also offer greater efficacy and tolerability than treatments of the 1990 's. These new therapies, particularly the newer immunotherapies including fingolimod, natalizumab, dimethyl fumarate, teriflunomide, alemtuzuma can yield long-lasting benefits, although greater risks. As well, Daclizumab and Ocrelizumab emerge as compounds for further treatments. Further research is needed and physicians and patients need to be carefully vigilant for signs of disease progression or side effects caused by the therapy.

\section{References}

1. Trapp BD and Nave KA: Multiple sclerosis: An immune or neurodegenerative disorder? Annu Rev Neurosci 31: 247-269, 2008.

2. Koch-Henriksen $\mathrm{N}$ and Sørensen PS: The changing demographic pattern of multiple sclerosis epidemiology. Lancet Neurol 9: 520-532, 2010 .

3. Kurtzke JF: Epidemiology of multiple sclerosis. Does this really point toward an etiology? Lectio doctoralis. Neurol Sci 21: 383-403, 2000.

4. Jersild C, Svejgaard A and Fog T: HL-A antigens and multiple sclerosis. Lancet 1: 1240-1241, 1972.

5. Farias AS and Santos LM: How can proteomics elucidate the complexity of multiple sclerosis? Proteomics Clin Appl 9: 844-847, 2015.

6. Oksenberg JR: Decoding multiple sclerosis: An update on genomics and future directions. Expert Rev Neurother 13 (Suppl 12): 11-19, 2013.

7. Magliozzi R, Howell O, Vora A, Serafini B, Nicholas R, Puopolo M, Reynolds R and Aloisi F: Meningeal B-cell follicles in secondary progressive multiple sclerosis associate with early onset of disease and severe cortical pathology. Brain 130: 1089-104, 2007.

8. Bjartmar C, Wujek JR and Trapp BD: Axonal loss in the pathology of MS: Consequences for understanding the progressive phase of the disease. J Neurol Sci 206: 165-171, 2003.

9. Celius EG and Smestad C: Change in sex ratio, disease course and age at diagnosis in Oslo MS patients through seven decades. Acta Neurol Scand (Suppl) 120: 27-29, 2009. 
10. de Sa JCC, Airas L, Bartholome E, Grigoriadis N, Mattle H, Oreja-Guevara C, O'Riordan J, Sellebjerg F, Stankoff B, Vass K, et al: Symptomatic therapy in multiple sclerosis: A review for a multimodal approach in clinical practice. Ther Adv Neurol Disord 4: 139-168, 2011.

11. Holland BE: Factors affecting quality of life in persons with multiple sclerosis (unpublished $\mathrm{PhD}$ thesis). The University of Texas at Arlington, 2014.

12. Charo IF and Ransohoff RM: The many roles of chemokines and chemokine receptors in inflammation. N Engl J Med 354: 610-621, 2006

13. Lublin FD and Reingold SC: Defining the clinical course of multiple sclerosis: results of an international survey. National Multiple Sclerosis Society (USA) Advisory Committee on Clinical Trials of New Agents in Multiple Sclerosis. Neurology 46: 907-911, 1996.

14. Coles AJ, Cox A, Le Page E, Jones J, Trip SA, Deans J, Seaman S, Miller DH, Hale G, Waldmann H, et al: The window of therapeutic opportunity in multiple sclerosis: Evidence from monoclonal antibody therapy. J Neurol 253: 98-108, 2006.

15. Feinstein A: The clinical neuropsychiatry of multiple sclerosis 2nd edition. Cambridge University Press, New York, NY, 2007

16. Compston A and Coles A: Multiple sclerosis. Lancet 372: $1502-1517,2008$

17. Lucchinetti C, Brück W, Parisi J, Scheithauer B, Rodriguez M and Lassmann $\mathrm{H}$ : Heterogeneity of multiple sclerosis lesions: Implications for the pathogenesis of demyelination. Ann Neurol 47: 707-717, 2000.

18. van der Valk P and De Groot CJ: Staging of multiple sclerosis (MS) lesions: Pathology of the time frame of MS. Neuropathol Appl Neurobiol 26: 2-10, 2000.

19. Mahad DH, Trapp BD and Lassmann H: Pathological mechanisms in progressive multiple sclerosis. Lancet Neurol 14: $183-193,2015$.

20. Kutzelnigg A, Lucchinetti CF, Stadelmann C, Brück W, Rauschka H, Bergmann M, Schmidbauer M, Parisi JE and Lassmann H: Cortical demyelination and diffuse white matter injury in multiple sclerosis. Brain 128: 2705-2712, 2005.

21. Milo R and Miller A: Revised diagnostic criteria of multiple sclerosis. Autoimmun Rev 13: 518-524, 2014

22. Glass CK, Saijo K, Winner B, Marchetto MC and Gage FH: Mechanisms underlying inflammation in neurodegeneration. Cell 140: 918-934, 2010.

23. Lassmann H, van Horssen J and Mahad D: Progressive multiple sclerosis: Pathology and pathogenesis. Nat Rev Neurol 8: 647-656, 2012.

24. Zindler E and Zipp F: Neuronal injury in chronic CNS inflammation. Best Pract Res Clin Anaesthesiol 24: 551-562, 2010.

25. Traugott U, Reinherz EL and Raine CS: Multiple sclerosis. Distribution of T cells, T cell subsets and Ia-positive macrophages in lesions of different ages. J Neuroimmunol 4: 201-221, 1983.

26. Ferguson B, Matyszak MK, Esiri MM and Perry VH: Axonal damage in acute multiple sclerosis lesions. Brain 120: 393-399, 1997.

27. Bitsch A, Schuchardt J, Bunkowski S, Kuhlmann T and Brück W: Acute axonal injury in multiple sclerosis. Correlation with demyelination and inflammation. Brain 123: 1174-1183, 2000.

28. Hu D, Ikizawa K, Lu L, Sanchirico ME, Shinohara ML and Cantor H: Analysis of regulatory CD8 T cells in Qa-1-deficient mice. Nat Immunol 5: 516-523, 2004.

29. Mars LT, Bauer J, Gross DA, Bucciarelli F, Firat H, Hudrisier D, Lemonnier F, Kosmatopoulos K and Liblau RS: CD8 T cell responses to myelin oligodendrocyte glycoprotein-derived peptides in humanized HLA-A*0201-transgenic mice. J Immunol 179: 5090-5098, 2007.

30. Schumacher GA, Beebe G, Kibler RF, Kurland LT, Kurtzke JF, McDowell F, Nagler B, Sibley WA, Tourtellotte WW and Willmon TL: Problems of experimental trials of therapy in multiple sclerosis: Report by the panel on the evaluation of experimental trials of therapy in multiple sclerosis. Ann N Y Acad Sci 122: 552-568, 1965.
31. Poser CM, Paty DW, Scheinberg L, McDonald WI, Davis FA, Ebers GC, Johnson KP, Sibley WA, Silberberg DH and Tourtellotte WW: New diagnostic criteria for multiple sclerosis: Guidelines for research protocols. Ann Neurol 13: 227-231, 1983.

32. Lublin FD, Reingold SC, Cohen JA, Cutter GR, Sørensen PS, Thompson AJ, Wolinsky JS, Balcer LJ, Banwell B, Barkhof F, et al: Defining the clinical course of multiple sclerosis: The 2013 revisions. Neurology 83: 278-286, 2014.

33. Calabresi PA, Kieseier BC, Arnold DL, Balcer LJ, Boyko A, Pelletier J, Liu S, Zhu Y, Seddighzadeh A, Hung S, et al; ADVANCE Study Investigators: Pegylated interferon $\beta-1 \mathrm{a}$ for relapsing-remitting multiple sclerosis (ADVANCE): A randomised, phase 3, double-blind study. Lancet Neurol 13 657-665, 2014.

34. Gold R, Kappos L, Arnold DL, Bar-Or A, Giovannoni G, Selmaj K, Tornatore C, Sweetser MT, Yang M, Sheikh SI, et al; DEFINE Study Investigators: Placebo-controlled phase 3 study of oral BG-12 for relapsing multiple sclerosis. N Engl J Med 367: 1098-1107, 2012

35. Fox RJ, Miller DH, Phillips JT, Hutchinson M, Havrdova E, Kita M, Yang M, Raghupathi K, Novas M, Sweetser MT, et al; CONFIRM Study Investigators: Placebo-controlled phase 3 study of oral BG-12 or glatiramer in multiple sclerosis. N Engl J Med 367: 1087-1097, 2012

36. Munger KL, Levin LI, Hollis BW, Howard NS and Ascherio A: Serum 25-hydroxyvitamin D levels and risk of multiple sclerosis JAMA 296: 2832-2838, 2006.

37. Ascherio A and Munger KL: Environmental risk factors for multiple sclerosis. Part I: The role of infection. Ann Neurol 61: 288-299, 2007.

38. Stein MS, Liu Y, Gray OM, Baker JE, Kolbe SC, Ditchfield MR, Egan GF, Mitchell PJ, Harrison LC, Butzkueven H, et al: A randomized trial of high-dose vitamin D2 in relapsing-remitting multiple sclerosis. Neurology 77: 1611-1618, 2011.

39. Mosayebi G, Ghazavi A, Ghasami K, Jand Y and Kokhaei P: Therapeutic effect of vitamin D3 in multiple sclerosis patients. Immunol Invest 40: 627-639, 2011.

40. Shaygannejad V, Janghorbani M, Ashtari F and Dehghan H: Effects of adjunct low-dose vitamin D on relapsing-remitting multiple sclerosis progression: preliminary findings of a randomized placebo-controlled trial. Mult Scler Int 2012: 452541,2012

41. Soilu-Hänninen M, Åivo J, Lindström B-M, Elovaara I, Sumelahti ML, Färkkilä M, Tienari P, Atula S, Sarasoja T, Herrala L, et al: A randomised, double blind, placebo controlled trial with vitamin D3 as an add on treatment to interferon $\beta-1 \mathrm{~b}$ in patients with multiple sclerosis. J Neurol Neurosurg Psychiatry 83: 565-571, 2012.

42. Chataway J, Schuerer N, Alsanousi A, Chan D, MacManus D, Hunter K, Anderson V, Bangham CR, Clegg S, Nielsen C, et al: Effect of high-dose simvastatin on brain atrophy and disability in secondary progressive multiple sclerosis (MS-STAT): A randomised, placebo-controlled, phase 2 trial. Lancet 383: 2213-2221, 2014

43. Ontaneda D and Fox RJ: Progressive multiple sclerosis. Curr Opin Neurol 28: 237-243, 2015

44. Bielekova B, Catalfamo M, Reichert-Scrivner S, Packer A, Cerna M, Waldmann TA, McFarland H, Henkart PA and Martin R: Regulatory CD56(bright) natural killer cells mediate immunomodulatory effects of IL-2Ralpha-targeted therapy (daclizumab) in multiple sclerosis. Proc Natl Acad Sci USA 103: 5941-5946, 2006.

45. Hauser SL, Waubant E, Arnold DL, Vollmer T, Antel J, Fox RJ, Bar-Or A, Panzara M, Sarkar N, Agarwal S, et al; HERMES Trial Group: B-cell depletion with rituximab in relapsing-remitting multiple sclerosis. N Engl J Med 358: 676-688, 2008.

46. Gold J, Goldacre R, Maruszak H, Giovannoni G, Yeates D and Goldacre M: HIV and lower risk of multiple sclerosis: beginning to unravel a mystery using a record-linked database study. J Neurol Neurosurg Psychiatry 86: 9-12, 2015. 